\title{
Duality, Ancestral and Diffusion Processes in Models with Selection*
}

\author{
Shuhei Mano ${ }^{\dagger}$ \\ Department of Statistics, University of Oxford, OX1 3TG, United Kingdom \\ Graduate School of Natural Sciences, Nagoya City University, Nagoya 467-8501, Japan \\ Japan Science and Technology Agency, 4-1-8 Honcho, Kawaguchi 332-0012, Japan
}

\footnotetext{
* Part of the research for this paper was carried out while the author visited the Department of Statistics at University of Oxford.

$\dagger$ The author was supported in part by Grants-in-Aids for Scientific Research from the Ministry of Education, Culture, Sports, Science and Technology of Japan.
}

Address for correspondence: Graduate School of Natural Sciences, Nagoya City University, 1 Yamanohata, Mizuho-cho, Mizuho-ku, Nagoya 467-8501, Japan;

E-mail: mano@nsc.nagoya-cu.ac.jp; Tel/Fax: +81-(0)52-872-5821 


\section{abstract}

The ancestral selection graph in population genetics was introduced by Krone and Neuhauser (1997) as an analogue of the coalescent genealogy of a sample of genes from a neutrally evolving population. The number of particles in this graph, followed backwards in time, is a birth and death process with quadratic death and linear birth rates. In this paper an explicit form of the probability distribution of the number of particles is obtained by using the density of the allele frequency in the corresponding diffusion model obtained by Kimura (1955). It is shown that the process of fixation of the allele in the diffusion model corresponds to convergence of the ancestral process to its stationary measure. The time to fixation of the allele conditional on fixation is studied in terms of the ancestral process.

Keywords: Ancestral Process, Diffusion Model, Ancestral Selection Graph, Coalescent Model, Birth and Death Process 


\section{INTRODUCTION}

In population genetics, the genealogy of a sample of genes plays an important role in a probabilistic description of the sample. Consider a discrete-time Wright-Fisher model of a population consisting of $2 N$ neutral genes. If we measure time in units of $2 N$ generations and let $N$ tend to infinity, then the Wright-Fisher process converges to a diffusion process. The genealogy of a sample of $n$ genes, followed backwards in time, is described by the coalescent process (Kingman, 1982b). The convergence is robust under a number of different models (e.g. Moran model). Let $\left\{a_{n}(t) ; t \geq 0\right\}$ be the number of ancestors of a sample of $n$ genes at time $t$ in the past. Then the size process $\left\{a_{n}(t) ; t \geq 0\right\}$ is a death process with death rate $i(i-1) / 2$ when the size is $i$. The size process will be referred to as the ancestral process. The distribution of $a_{n}(t)$ is known (see Griffiths (1979), Tavaré (1984)) and

$$
\mathbb{P}\left[a_{n}(t)=i\right]=\sum_{k=i}^{n} \frac{(-1)^{k-i}(2 k-1)(i)_{k-1}[n]_{k}}{i !(k-i) !(n)_{k}} \rho_{k}^{0}(t), \quad i=1,2, \ldots, n,
$$

where $\rho_{k}^{0}(t):=\exp \{-k(k-1) t / 2\},[n]_{k}=n(n-1) \cdots(n-k+1)$ and $(n)_{k}=n(n+1) \cdots(n+$ $k-1)$. The total variation norm between $a_{n}(t)$ and $a_{n}(\infty)=\delta_{1}$ has a simple form

$$
\left\|a_{n}(t), \delta_{1}\right\|_{\text {var }}=1-\mathbb{P}\left[a_{n}(t)=1\right] .
$$

There is a first time $W_{n, 1}^{0}$ such that $a_{n}\left(W_{n, 1}^{0}\right)=1$, which is the time to the most recent common ancestor. The density of $W_{n, 1}^{0}$ follows

$$
\mathbb{P}\left[W_{n, 1}^{0} \leq t\right]=\mathbb{P}\left[a_{n}(t)=1\right]=\sum_{k=1}^{n} \frac{(-1)^{k-1}(2 k-1)[n]_{k}}{(n)_{k}} \rho_{k}^{0}(t) .
$$

A bound for $\mathbb{P}\left[a_{n}(t)=1\right]$ is known (see Kingman (1982b), Tavaré (1984)) and

$$
e^{-t} \leq 1-\mathbb{P}\left[a_{n}(t)=1\right] \leq 3 \frac{n-1}{n+1} e^{-t}, \quad n=2,3, \ldots
$$

The ancestral selection graph introduced by Krone and Neuhauser (1997) is an analogue of the coalescent genealogy. Assume that a pair of allelic types $A_{1}$ and $A_{2}$ are segregating in a population, and the selective advantage of a type $A_{1}$ gene over a type $A_{2}$ gene is 
$s(>0)$. Let $N \rightarrow \infty$ while $c=N s$ is held constant. The elements are referred to as particles. Let $b_{n}(t)$ be the number of edges, or ancestral particles, in a cross section of the ancestral selection graph for a sample of $n$ genes at time $t$ in the past. In the ancestral selection graph, coalescence occurs at rate $\alpha_{i}=i(i-1) / 2$ and branching occurs at rate $\beta_{i}=2 c i$ when the size is $i$. Then the ancestral process $\left\{b_{n}(t) ; t \geq 0\right\}$ is a birth and death process with rates $\beta_{i}$ and $\alpha_{i}$. A particle is called real if it is a part of the real genealogy of the sample, otherwise the particle is called virtual. If two particles reach a coalescing point, the resulting particle is real if and only if at least one of the two particles is real, otherwise the resulting particle is virtual. If a real particle reaches a branching point, it splits into a real particle and into a virtual particle. If a virtual particle reaches a branching point, it splits into two virtual particles. If a type $A_{2}$ particle reaches a branching point, it splits into two type $A_{2}$ particles. If a type $A_{1}$ particle reaches a branching point, it splits into two particles, where at least one of the two particles is type $A_{1}$. Because the death rates are quadratic while the the birth rates are only linear, there is a first time $W_{n, 1}^{c}$ such that $b_{n}\left(W_{n, 1}^{c}\right)=1$. Krone and Neuhauser (1997) consider stopping the process at this time, since the genetic composition of the sample is determined by then. They called the ancestral particle at the time the ultimate ancestor. In the case of no mutation, the real genealogy embedded in an ancestral selection graph is the same as in the neutral process (Krone and Neuhauser (1997); Theorem 3.12). Thus the ancestral process of the real particles can be described by the neutral process $\left\{a_{n}(t) ; t \geq 0\right\}$. In this article, we discuss properties of the ancestral process $\left\{b_{n}(t) ; t \geq 0\right\}$ without mutation which is not stopped upon reaching the ultimate ancestor. Fearnhead (2002) has studied a process which is not stopped upon reaching the ultimate ancestor. He identifies the stationary distribution of the process and uses the distribution to characterize the substitution process to the common ancestor.

Kimura (1955) studied the density of the allele frequency by analyzing the diffusion process to which the Wright-Fisher model with directional selection converges. Let $x_{p}(t)$ 
be the frequency of the allele $A_{1}$ at time $t$ forward in a population in which the initial frequency is $p$. Then the Kolmogorov forward equation for the diffusion process $\left\{x_{p}(t) ; t \geq\right.$ $0\}$ on $(0,1)$ is

$$
\frac{\partial \phi}{\partial t}=\frac{1}{2} \frac{\partial^{2}}{\partial x^{2}}\{x(1-x) \phi\}-2 c \frac{\partial}{\partial x}\{x(1-x) \phi\},
$$

with the initial condition $\phi(p, x ; 0)=\delta(x-p)$. The solution is

$$
\phi(p, x ; t)=2\left(1-r^{2}\right) e^{c(r-1)} e^{2 c x} \sum_{k=0}^{\infty} \frac{V_{1 k}^{(1)}(c, r) V_{1 k}^{(1)}(c, z)}{N_{1 k}} \rho_{k+2}^{c}(t),
$$

where $r=1-2 p, z=1-2 x, \rho_{k+2}^{c}(t):=\exp \left(-\lambda_{k} t\right), k=0,1,2, \ldots$ and $-\lambda_{k}\left(0<\lambda_{0}<\right.$ $\left.\lambda_{1}<\cdots\right)$ are the eigenvalues of the generator. A plot of $\lambda_{0}$ is given in Figure 1 of Kimura (1955). $V_{1 k}^{(1)}(c, z)$ is the oblate spheroidal wave function (see Appendix):

$$
V_{1 k}^{(1)}(c, z)=\sum_{l \geq 0}^{\prime} f_{l}^{k}(c) T_{l}^{1}(z)
$$

where $T_{l}^{1}(z)$ is the Gegenbauer function (may also be denoted by $C_{l}^{\frac{3}{2}}(z)$ ) and the summation is over even values of $l$ if $k$ is even, odd values of $l$ if $k$ is odd. $N_{1 k}$ is the normalization constant of $V_{1 k}^{(1)}(c, z)$. The probability mass at the exit boundaries are

$$
f(p, 1 ; t)=2\left(1-r^{2}\right) e^{c(r+1)} \sum_{k=0}^{\infty} \frac{V_{1 k}^{(1)}(c, r) V_{1 k}^{(1)}(c,-1)}{2 \lambda_{k} N_{1 k}}\left(1-\rho_{k+2}^{c}(t)\right),
$$

and

$$
f(p, 0 ; t)=2\left(1-r^{2}\right) e^{c(r-1)} \sum_{k=0}^{\infty} \frac{V_{1 k}^{(1)}(c, r) V_{1 k}^{(1)}(c, 1)}{2 \lambda_{k} N_{1 k}}\left(1-\rho_{k+2}^{c}(t)\right) .
$$

In Section 2, the ancestral process $\left\{b_{n}(t) ; t \geq 0\right\}$ without absorbing states is studied. An explicit form of the probability distribution of $b_{n}(t)$ is obtained by using the moment duality between the ancestral process and the Wright-Fisher diffusion with directional selection. It is also observed that the distribution of the ancestral process converges to a stationary distribution. In Section 3, the rate of convergence is discussed. In contrast to the neutral process, the final rates of convergence are given by the largest eigenvalue for all the states. Bounds for the probability that $b_{n}(t)$ is at the state 1 are obtained by an 
elementary martingale argument, which corresponds to the bounds (1.4) for the neutral process. In Section 4, the ancestral process with absorbing states is considered. It is shown that the first passage times of the ancestral process $\left\{b_{n}(t) ; t \geq 0\right\}$ at the states $1,2, \ldots, n-1$ are larger than that in the neutral process for all the states. By killing the modified process, in which the state 1 is the absorbing state, the form of the joint probability generating function of $b_{n}(t)$ and the number of branching events is obtained. By using this formula, the expectation of the total length of the edges in the ancestral selection graph is obtained. In Section 5 , the ancestral process of the whole population $\left\{b_{\infty}(t) ; t \geq 0\right\}$ is studied. It is shown that the process of fixation of the allele in the diffusion model corresponds to convergence of the ancestral process to its stationary measure. The time to fixation of an allele conditional on fixation is studied in terms of the ancestral process. It is shown that the density of the time to fixation of a single mutant gene conditional on fixation is given by the probability of the whole population being descended from a single real ancestral particle, regardless of the allelic type. In the neutral process, the density of the waiting time until the ancestral process hits the state 1 and the density of the conditional fixation time are given by the probability that the ancestral process is at the state 1 . The property does not hold in the process with selection.

\section{Number of ANCESTRAl PARTiCles}

In this section, we will obtain an equation that relates the moments of the WrightFisher diffusion with directional selection to a Markov process that specifies the number of particles (real and virtual) that are present in the ancestral selection graph. To derive this result, we will exploit the concept of duality from the theory of Markov processes (Ethier and Kurtz (1986); Section 4.4). If $X=\left\{X_{t} ; t \geq 0\right\}$ and $Y=\left\{Y_{t} ; t \geq 0\right\}$ are Markov processes with values in sets $Z_{X}$ and $Z_{Y}$, respectively, then $X$ and $Y$ are said to be dual with respect to a function $f(x, y)$ if the identity

$$
\mathbb{E}_{x}\left[f\left(X_{t}, y\right)\right]=\mathbb{E}_{y}\left[f\left(x, Y_{t}\right)\right]
$$


holds for every $x \in Z_{X}$ and $y \in Z_{Y}$. Duality is a useful concept because it allows us to use our knowledge of one process to learn about the other. Although there is no general procedure for identifying dual processes, duality can sometimes be deduced using simple generator calculations. Specifically, if $G_{x}$ is the infinitesimal generator of the process $X$ and $G_{y}$ is the infinitesimal generator of the process $Y$, then the duality relationship shown in (2.1) will be satisfied if the identity

$$
G_{x} f(x, y)=G_{y} f(x, y)
$$

holds for all $x$ and $y$. Here we think $G_{x} f(x, y)$ as acting on the $x$-variable of the function $f(x, y)$ for each fixed value of $y$.

To apply these results to the Wright-Fisher diffusion with selection, it will be necessary to consider the frequency $y_{q}(t)=1-x_{p}(t)(q=1-p)$ of the less fit allele, which is itself governed by a Wright-Fisher diffusion with generator:

$$
G_{y} f(y)=\frac{1}{2} y(1-y) f^{\prime \prime}(y)-2 c y(1-y) f^{\prime}(y)
$$

Notice that the selection coefficient is negative in this case $(c \geq 0)$. If we define the function $f(y, n)=y^{n}$, then a simple calculation shows that

$$
\begin{aligned}
G_{y} f(y, n) & =\left(\begin{array}{l}
n \\
2
\end{array}\right)[f(y, n-1)-f(y, n)]+2 c n[f(y, n+1)-f(y, n)] \\
& =G_{n} f(y, n),
\end{aligned}
$$

where $G_{n}$ is the operator defined by

$$
G_{n} f(n)=\left(\begin{array}{l}
n \\
2
\end{array}\right)[f(n-1)-f(n)]+2 c n[f(n+1)-f(n)] .
$$

In other words, $G_{n}$ is the infinitesimal generator of the birth and death process $\left\{b_{n}(t) ; t \geq\right.$ $0\}$ which keeps track of the number of ancestral particles in the ancestral selection graph. Because $f(y, n)$ is bounded, we can use a result of Ethier and Kurtz (1986) (Chapter 4, Corollary 4.13) to deduce that the Wright-Fisher diffusion $\left\{y_{q}(t) ; t \geq 0\right\}$ and the birth and death process $b_{n}(t)$ are dual with respect to the function $f(y, n)$ : 


\section{Theorem 2.1.}

$$
\mathbb{E}\left[q^{b_{n}(t)}\right]=\mathbb{E}\left[\left(y_{q}(t)\right)^{n}\right], \quad n=1,2, \ldots
$$

Because the right-hand side of this identity involves moments of the process $\left\{y_{q}(t) ; t \geq\right.$ $0\}$, the process $\left\{b_{n}(t) ; t \geq 0\right\}$ is said to be a moment dual for $\left\{y_{q}(t) ; t \geq 0\right\}$. The existence of moment duals of Wright-Fisher diffusions with polynomial coefficients was first shown by Shiga (1981), and the explicit description of duality between the birth and death process and the Wright-Fisher diffusion with directional selection was discussed by Athreya and Swart (2005). The duality can be proved in terms of the ancestral selection graph (Athreva and Swart (2005)):

Proof. Partition an ancestral selection graph $\mathcal{G}$ into disconnected subgraphs $\mathcal{G}_{i}, i=1,2, \ldots$ Let $\mathcal{E}_{t}$ be the edges, or the ancestral particles, of a cross section of $\mathcal{G}$ taken at time $t$ backward. Then, $b_{n}(t)=\left|\mathcal{E}_{t}\right|$. Each $\mathcal{E}_{0} \cap \mathcal{G}_{i}$ consists only of type $A_{2}$ particles if and only if $\mathcal{E}_{t} \cap \mathcal{G}_{i}$ consists only of type $A_{2}$ particles, since at least one type $A_{1}$ particle survives from time $t$ to 0 if $\mathcal{E}_{t} \cap \mathcal{G}_{i}$ contain type $A_{1}$ particles. Here the ancestral selection graph is viewed forward in time. If a type $A_{1}$ particle reaches a coalescence point, the number of type $A_{1}$ particles increase by 1 . If a type $A_{1}$ particle reaches a branching point and meets another particle, the resulting particle is always type $A_{1}$. Thus, a sample consists only of type $A_{2}$ particles if and only if the ancestral particles at time $t$ consists only of type $A_{2}$ particles.

If a sample contains type $A_{1}$ particles, then for $n=0,1, \ldots ; m=1,2, \ldots$,

$$
\mathbb{E}\left[\left(x_{p}(t)\right)^{m}\left(y_{q}(t)\right)^{n}\right]=\mathbb{E}\left[\left(1-y_{q}(t)\right)^{m}\left(y_{q}(t)\right)^{n}\right]=\sum_{i=0}^{m}(-1)^{i}\left(\begin{array}{c}
m \\
i
\end{array}\right) \mathbb{E}\left[q^{b_{i+n}(t)}\right],
$$

with the convention $b_{0}(t)=0$. In particular,

$$
\mathbb{E}\left[x_{p}(t)\right]=\mathbb{E}\left[1-y_{q}(t)\right]=\mathbb{E}\left[1-q^{b_{1}(t)}\right]=\sum_{k=1}^{\infty} \mathbb{P}\left[b_{1}(t)=k\right] \sum_{i=1}^{k}\left(\begin{array}{c}
k \\
i
\end{array}\right) p^{i} q^{k-i}
$$


The expression follows immediately from the distribution of the number of particles in $\mathcal{E}_{t}$. Since the ancestral selection graph is irreducible, a type $A_{1}$ particle is sampled if and only if $\mathcal{E}_{t}$ contains at least one type $A_{1}$ particle. The first summation is over $\left|\mathcal{E}_{t}\right|$ and the second summation is over the number of type $A_{1}$ particles.

By the Itô formula, we have a system of differential equations for the moments of $y_{q}(t)$

$$
\frac{d \xi_{n}}{d t}=-\left(\alpha_{n}+\beta_{n}\right) \xi_{n}+\alpha_{n} \xi_{n-1}+\beta_{n} \xi_{n+1}, \quad n=1,2, \ldots
$$

where $\xi_{n}=\mathbb{E}\left[\left(y_{q}(t)\right)^{n}\right]$. The Kolmogorov backward equation for the ancestral process $\left\{b_{n}(t) ; t \geq 0\right\}$ without absorbing states is also given by (2.9), where $\xi_{n}=\mathbb{P}\left[b_{n}(t)=i\right]$. Thus, (2.9) with $\xi_{n}=\mathbb{E}\left[q^{b_{n}(t)}\right]$ holds. The isomorphism of these equations is a consequence of $(\underline{2.6})$.

A realization of the ancestral selection graph consists of two disconnected subgraphs embedded in a diagram of a sample path of $x_{p}(t)$ can be seen in Figure 1. The abscissa is the forward time interval $(0, t)$ and ordinate is $x_{p}(t)$. Thick lines represent the real genealogy. Lines used by type $A_{2}$ particles are dotted. The graph contributes to $\mathbb{E}\left[\left(x_{p}(t)\right)^{3} y_{q}(t)\right]$ and $b_{4}(t)=4$. If an ancestral selection graph consists of the upper subgraph only, it contributes to $\mathbb{E}\left[y_{q}(t)\right]=\mathbb{E}\left[q^{b_{1}(t)}\right]$ and $b_{1}(t)=2$.

Using an integral transform by the Gegenbauer function (Erdélyi et al. (1954)) for $l=$ $0,1, \ldots ; n=1,2, \ldots ; i=0,1, \ldots$,

$$
\int_{-1}^{1} T_{l}^{1}(z)(1+z)^{n}(1-z)^{i} d z=\frac{2^{n+i} i !(l+1)(l+2)}{(n+1)_{i+1}}{ }_{3} F_{2}(-l, l+3, i+1 ; 2, i+n+2 ; 1)
$$

where ${ }_{3} F_{2}(-l, l+3, i+1 ; 2, i+n+2 ; 1)$ is the generalized hypergeometric function, and with an identity (5.2), it is possible to obtain an explicit expression for the probability 
generating function of $b_{n}(t)$, and we have

$$
\begin{aligned}
\mathbb{E}\left[q^{b_{n}(t)}\right] & =\mathbb{E}\left[y_{q}(t)^{n}\right]=\int_{0}^{1}(1-x)^{n} \phi(x, p ; t) d x+f(p, 0 ; t) \\
& =\frac{e^{4 c q}-1}{e^{4 c}-1}+2\left(1-r^{2}\right) e^{c(r-1)} \sum_{k=0}^{\infty} \frac{V_{1 k}^{(1)}(c, r)}{N_{1 k}}\left\{F_{k n}(c)-\frac{V_{1 k}^{(1)}(c, 1)}{2 \lambda_{k}}\right\} \rho_{k+2}^{c}(t) \\
& =\sum_{i=1}^{\infty} \mathbb{P}\left[b_{n}(t)=i\right] q^{i},
\end{aligned}
$$

where

$$
F_{k n}(c):=\sum_{l \geq 0}^{\prime} f_{l}^{k}(c) \sum_{i=0}^{\infty} \frac{(2 c)^{i}}{(n+1)_{i+1}} \sum_{j=0}^{l} \frac{(-l)_{j}(l+1)_{j+2}(i+1)_{j}}{2 \cdot j !(j+1) !(i+n+2)_{j}}
$$

$r=1-2 p$ and $f(p, 0 ; t)$ is the probability mass at 0 given in (1.9). If $n=\infty, F_{k \infty}(c)=0$ and $f(p, 0 ; t)$ gives the probability generating function. Using a power series expansion in $q$ of the Gegenbauer function

$$
T_{l}^{1}(r)=(-1)^{l} \sum_{i=0}^{l} \frac{(-l)_{i}(l+1)_{i+2}}{2 \cdot i !(i+1) !} q^{i}, \quad l=0,1, . .
$$

we obtain an explicit expression for the probability distribution of $b_{n}(t)$ :

$$
\mathbb{P}\left[b_{n}(t)=1\right]=\pi_{1}+8 e^{-2 c} \sum_{k=0}^{\infty} \frac{V_{1 k}^{(1)}(c,-1)}{N_{1 k}}\left\{F_{k n}(c)-\frac{V_{1 k}^{(1)}(c, 1)}{2 \lambda_{k}}\right\} \rho_{k+2}^{c}(t),
$$

and

$$
\mathbb{P}\left[b_{n}(t)=i\right]=\pi_{i}+8 e^{-2 c} \sum_{k=0}^{\infty} \frac{G_{k i}(c)}{N_{1 k}}\left\{F_{k n}(c)-\frac{V_{1 k}^{(1)}(c, 1)}{2 \lambda_{k}}\right\} \rho_{k+2}^{c}(t), \quad i=2,3, \ldots
$$

where

$$
\begin{aligned}
G_{k i}(c):= & \sum_{l \geq i-1}{ }^{\prime} f_{l}^{k}(c)(-1)^{l} \frac{(-l)_{i-1}(l+1)_{i+1}}{2(i-1) ! i !} \\
& +\sum_{j=1}^{i-1} \frac{(2 c)^{j-1}(2 c-j)}{j \cdot(j-1) !} \sum_{l \geq i-j-1}{ }^{\prime} f_{l}^{k}(c)(-1)^{l} \frac{(-l)_{i-j-1}(l+1)_{i-j+1}}{2(i-j-1) !(i-j) !}
\end{aligned}
$$


and $\pi_{i}$ are given in (3.1). Note that there are finite probabilities at the states $n+1, n+2, \ldots$. The expected number of ancestral particles is

$$
\begin{aligned}
\mathbb{E}\left[b_{n}(t)\right]= & \pi_{1} e^{4 c}+8 e^{-2 c} \sum_{k=0}^{\infty} \frac{V_{1 k}^{(1)}(c,-1)}{N_{1 k}}\left\{F_{k n}(c)-\frac{V_{1 k}^{(1)}(c, 1)}{2 \lambda_{k}}\right\} \rho_{k+2}^{c}(t) \\
& +8 e^{-2 c} \sum_{i=2}^{\infty} i \sum_{k=0}^{\infty} \frac{G_{k i}(c)}{N_{1 k}}\left\{F_{k n}(c)-\frac{V_{1 k}^{(1)}(c, 1)}{2 \lambda_{k}}\right\} \rho_{k+2}^{c}(t),
\end{aligned}
$$

and the falling factorial moments are

$$
\mathbb{E}\left[\left[b_{n}(t)\right]_{i}\right]=i ! \pi_{i} e^{4 c}+8 e^{-2 c} \sum_{j=i}^{\infty}[j]_{i} \sum_{k=0}^{\infty} \frac{G_{k j}(c)}{N_{1 k}}\left\{F_{k n}(c)-\frac{V_{1 k}^{(1)}(c, 1)}{2 \lambda_{k}}\right\} \rho_{k+2}^{c}(t), \quad i=2,3, \ldots
$$

For small $c$, the probability distribution is approximately

$$
\mathbb{P}\left[b_{n}(t)=1\right]=\mathbb{P}\left[a_{n}(t)=1\right]-2 c+2 c \sum_{k=2}^{n+1}(-1)^{k}(2 k-1)\left\{\frac{[n]_{k}}{(n)_{k}}+\frac{k(k-1)[n]_{k-1}}{(n)_{k+1}}\right\} \rho_{k}^{0}(t)+O\left(c^{2}\right),
$$

and for $i=2,3, \ldots$,

$$
\begin{aligned}
\mathbb{P}\left[b_{n}(t)=i\right]= & \mathbb{P}\left[a_{n}(t)=i\right]+2 c \delta_{i, 2}-2 c \sum_{k=i}^{n+1} \frac{(-1)^{k-i}(2 k-1)(i)_{k-1}}{i !(k-i) !}\left\{\frac{k(k-1)[n]_{k-1}}{(n)_{k+1}}\right. \\
& \left.+\frac{\left(k^{2}-k+2 i-2\right)[n]_{k}}{(k-i+1)(k+i-2)(n)_{k}}\right\} \rho_{k}^{0}(t)+2 c \frac{(i-1)_{i-1}[n]_{i-1}}{(i-1) !(n)_{i-1}} \rho_{i-1}^{0}(t)+O\left(c^{2}\right),
\end{aligned}
$$

with a convention $[n]_{n+1}=0$, where $a_{n}(t)$ is the number of ancestors of a sample of $n$ neutral genes at the time $t$ in the past.

It is possible to obtain the solution of (2.9) as a perturbation series in $2 c$, where the series is represented by eigenvalues of the neutral process. Let

$$
\xi_{n}=\xi_{n}^{(0)}+2 c \xi_{n}^{(1)}+(2 c)^{2} \xi_{n}^{(2)}+\cdots, \quad n=1,2, \ldots
$$

Denote the rate matrix of the neutral process $\left\{a_{n}(t) ; t \geq 0\right\}$ by $Q_{0}=\left(q_{0, i j}\right)$, where $q_{0, i+1, i}=\alpha_{i+1}, q_{0, i i}=-\alpha_{i}$ for $i=1,2, \ldots$ and other elements are zero. Let the Laplace transform of $\xi_{n}^{(i)}(t)$ be $\nu_{n}^{(i)}(\lambda)$. It is straightforward to show that

$$
\nu^{(i)}=\left\{\left(Q_{0}-\lambda E\right)^{-1} C\right\}^{i}\left(\lambda E-Q_{0}\right)^{-1} \xi^{(0)}(0), \quad i=1,2, \ldots
$$


where $C=\left(c_{i j}\right)$ is given by $c_{i i}=i, c_{i, i+1}=-i$ for $i=1,2, \ldots$ and other elements are zero. Note that the inverse Laplace transform of the element in the $n$-th row and $i$-th column of the matrix $\left\{\left(Q_{0}-\lambda E\right)^{-1} C\right\}^{j}\left(\lambda E-Q_{0}\right)^{-1}$ gives the $j$-th order coefficients in $2 c$ of $\mathbb{P}\left[b_{n}(t)=i\right]$.

Let $r_{n}(t)$ be the number of branching events in the time interval $(0, t)$ in the ancestral selection graph of a sample of $n$ genes, where $r_{n}(0)=0$. The joint probability generating functions of $b_{n}(t)$ and $r_{n}(t)$ satisfy a system of differential equation

$$
\frac{d \xi_{n}}{d t}=-\left(\alpha_{n}+v \beta_{n}\right) \xi_{n}+\alpha_{n} \xi_{n-1}+v \beta_{n} \xi_{n+1}-(1-v) \beta_{n} \xi_{n}, \quad n=1,2, . .
$$

with the initial condition $\xi_{n}(0)=q^{n}$, where $\xi_{n}=\mathbb{E}\left[q^{b_{n}(t)} v^{r_{n}(t)}\right]$. The solution is given by killing of the modified process $\left\{\tilde{b}_{n}(t) ; t \geq 0\right\}$ in which the selection coefficient is $v c$, and we have

$$
\mathbb{E}\left[q^{b_{n}(t)} v^{r_{n}(t)}\right]=\mathbb{E}\left[q^{\tilde{b}_{n}(t)} \exp \left\{-2 c(1-v) \int_{0}^{t} \tilde{b}_{n}(u) d u\right\}\right]
$$

By setting $v=0$ in the last expression, we obtain the identity

$$
\mathbb{E}\left[q^{b_{n}(t)}, r_{n}(t)=0\right]=\mathbb{E}\left[q^{a_{n}(t)} \exp \left\{-2 c \int_{0}^{t} a_{n}(u) d u\right\}\right]
$$

which shows the Poisson nature of the branching in the ancestral process $\left\{b_{n}(t) ; t \geq 0\right\}$. The integral is the total length of the edges in the neutral genealogy without branching in $(0, t)$. In particular, $\mathbb{P}\left[b_{1}(t)=1, r(t)=0\right]=e^{-2 c t}$.

\section{Convergence}

Standard results on birth and death processes (see, e.g., Karlin and Taylor (1975)) gives the stationary measure of the ancestral process $\left\{b_{n}(t) ; t \geq 0\right\}$. It is straightforward to obtain the stationary measure

$$
\pi_{i}:=\mathbb{P}\left[b_{n}(\infty)=i\right]=\frac{(4 c)^{i}}{i !\left(e^{4 c}-1\right)}, \quad i=1,2, \ldots
$$


which is the zero-truncated Poisson distribution. Since the ancestral process of the real particles is the neutral process $\left\{a_{n}(t) ; t \geq 0\right\}$ and the number of real particles becomes 1 in finite time, $\pi_{1}$ is the probability that there are no virtual particles.

It is clear from (2.13) and (2.14) that for $i=1,2 \ldots$,

$$
\pi_{i}-\mathbb{P}\left[b_{n}(t)=i\right]=O\left(\rho_{2}^{c}(t)\right), \quad t \rightarrow \infty .
$$

For small $c$, the final rates of convergence are approximately

$\lim _{t \rightarrow \infty}\left(\rho_{2}^{c}(t)\right)^{-1}\left\{\pi_{1}-\mathbb{P}\left[b_{n}(t)=1\right]\right\}=3 e^{-2 c}\left\{\frac{n-1}{n+1}-\frac{4 c}{(n+1)(n+2)}\right\}-2 c e^{-2 c} \delta_{n, 1}+O\left(c^{2}\right)$,

$\lim _{t \rightarrow \infty}\left(\rho_{2}^{c}(t)\right)^{-1}\left\{\pi_{2}-\mathbb{P}\left[b_{n}(t)=2\right]\right\}=-3 e^{-2 c}\left\{\frac{n-1}{n+1}-\frac{2 n(n-1) c}{n+2}\right\}-2 c e^{-2 c} \delta_{n, 1}+O\left(c^{2}\right)$,

and

$$
\lim _{t \rightarrow \infty}\left(\rho_{2}^{c}(t)\right)^{-1}\left\{\pi_{i}-\mathbb{P}\left[b_{n}(t)=i\right]\right\}=-3 e^{-2 c} \frac{n-1}{n+1} \frac{(2 c)^{i-2}}{(i-2) !}+O\left(c^{i-1}\right), \quad i=3,4, \ldots
$$

In contrast to the neutral process, the final rates of convergence are given by the largest eigenvalue for all the states. In the neutral process, we have

$$
\lim _{t \rightarrow \infty}\left(\rho_{i}^{0}(t)\right)^{-1} \mathbb{P}\left[a_{n}(t)=i\right]=\frac{(i)_{i}[n]_{i}}{i !(n)_{i}}, \quad i=1,2, \ldots, n
$$

The total variation norm has no simple form as in (1.2).

A simple argument gives a bound for $\mathbb{P}\left[b_{n}(t)=1\right]$. The event that the number of ancestral particles is 1 is a subset of the event that the number of real particle is 1 , and we have

$$
\mathbb{P}\left[b_{n}(t)=1\right] \leq \mathbb{P}\left[a_{n}(t)=1\right], \quad n=1,2, \ldots
$$

An elementary argument on a martingale gives explicit bounds for $\mathbb{P}\left[b_{n}(t)=1\right]$, which corresponds to the bounds (1.4) in the neutral process. Let $\eta(n ; c)$ satisfy a recursion

$$
\left(\lambda_{0}-\alpha_{n}-\beta_{n}\right) \eta(n ; c)+\alpha_{n} \eta(n-1 ; c)+\beta_{n} \eta(n+1 ; c)=0, \quad n=1,2, \ldots
$$


with the boundary condition $\eta(1 ; c)=-2 c$. Since $\eta$ is an eigenvector of the transition probability matrix of the ancestral process $\left\{b_{n}(t) ; t \geq 0\right\}, \eta\left(b_{n}(t) ; c\right)\left(\rho_{2}^{c}(t)\right)^{-1}$ is a martingale to the ancestral process (see, e.g., Karlin and Taylor (1975)). Then,

$$
\mathbb{E}\left[\eta\left(b_{n}(t) ; c\right)\right]=\eta(n ; c) \rho_{2}^{c}(t)
$$

Although the explicit form of $\eta(n ; c)$ is not available, it is possible to obtain an asymptotic form. Because of

$$
\frac{\eta(n ; c)}{\eta(n-1 ; c)} \rightarrow 1+\frac{2 \lambda_{0}}{n(n-1)}+O\left(n^{-3}\right), \quad n \rightarrow \infty
$$

we deduce the asymptotic form $\eta(n ; c) \approx \eta(\infty ; c)\left(1-2 \lambda_{0} / n\right)$, where $\eta(\infty ; c)$ is a function of $c$. Although the explicit form of $\eta(\infty ; c)$ is not available, it is very close to $3 \exp (-c)$ (See Figure 2).

For small $c, \eta(n ; c)$ can be expanded into a power series in $c$ :

$$
\eta(n ; c)=3 \frac{n-1}{n+1}\left\{1-\frac{n^{2}+n+2}{(n-1)(n+2)} c+\frac{4\left(3 n^{2}+10 n+18\right)}{25(n+2)(n+3)} c^{2}\right\}+O\left(c^{3}\right), \quad n=2,3, \ldots
$$

Note that $\eta(\infty ; c)$ is not exactly equal to $3 \exp (-c)$.

Lemma 3.1. $\eta(n ; c)$ is monotonically increasing in $n$.

Proof. Denote the rate matrix of the ancestral process $\left\{b_{n}(t) ; t \geq 0\right\}$ by $Q_{c}=\left(q_{c, i j}\right)$, where $q_{c, i+1, i}=\alpha_{i+1}, q_{c, i i}=-\left(\alpha_{i}+\beta_{i}\right), q_{c, i, i+1}=\beta_{i}$ for $i=1,2, \ldots$ and other elements are zero. $\eta$ is an eigenvector of an oscillatory matrix $E+Q_{c}(2 N)^{-1}$ which belongs to the second largest eigenvalue $1-\lambda_{0}(2 N)^{-1}$. An eigenvector of an oscillatory matrix which belongs to the second largest eigenvalue has exactly one variation of sign in the coordinates (see, Gantmacher (1959), pp. 105). Assume $\eta(i ; c)>0, i \geq L$ and $\eta(i ; c) \leq 0,1 \leq i \leq L-1$. 
Suppose $l \geq L-1$. By an induction we deduce from (3.8) that

$$
\begin{aligned}
\eta(l+1 ; c)-\eta(l ; c) & =\frac{\alpha_{l}}{\beta_{l}}\{\eta(l ; c)-\eta(l-1 ; c)\}-\lambda_{0} \eta(l ; c) \\
& =\frac{(l-1) !}{(4 c)^{l-1}}\left\{\eta(2 ; c)-\eta(1 ; c)-\frac{\lambda_{0}}{\pi_{2}} \sum_{i=2}^{l} \eta(i ; c) \pi_{i}\right\} .
\end{aligned}
$$

By taking $t=\infty$ in (3.9) it follows that

$$
\sum_{i=1}^{\infty} \eta(i ; c) \pi_{i}=0
$$

Thus,

$$
\eta(l+1 ; c)-\eta(l ; c)=\frac{\lambda_{0}}{8 c^{2} \pi_{l-1}} \sum_{i=l+1}^{\infty} \eta(i ; c) \pi_{i}>0 .
$$

Next, suppose $2 \leq l \leq L-2$. We have

$$
\eta(l+1 ; c)-\eta(l ; c)=\frac{(l-1) !}{(4 c)^{l-1}}\left\{\eta(2 ; c)-\eta(1 ; c)-\frac{\lambda_{0}}{\pi_{2}} \sum_{i=2}^{l} \eta(i ; c) \pi_{i}\right\}>0 .
$$

Finally, $\eta(2 ; c)-\eta(1 ; c)=\lambda_{0}>0$.

From Lemma 3.1 it follows that

$$
\mathbb{P}\left[b_{n}(t)=1\right] \eta(1 ; c)+\mathbb{P}\left[b_{n}(t)>1\right] \eta(2 ; c) \leq \mathbb{E}\left[\eta\left(b_{n}(t) ; c\right)\right]
$$

$$
\leq \mathbb{P}\left[b_{n}(t)=1\right] \eta(1 ; c)+\mathbb{P}\left[b_{n}(t)>1\right] \eta(\infty ; c),
$$

here we note that there are finite probabilities at the states larger than $n$. Then, from (3.9) we have the following bounds:

Theorem 3.2. If $\eta(n ; c)$ satisfies the recursion (3.8), then

$$
\frac{\eta(n ; c) e^{-\lambda_{0} t}+2 c}{\eta(\infty ; c)+2 c} \leq 1-\mathbb{P}\left[b_{n}(t)=1\right] \leq \frac{\eta(n ; c) e^{-\lambda_{0} t}+2 c}{\lambda_{0}}, \quad n=1,2, \ldots
$$

Figure 3 shows the bounds when $c=0,0.1,1$ and 8 for $n=10$. When $c>0$, in contrast to the neutral case, the states larger than 1 have finite probabilities in the stationary measure (3.1) and the upper and lower bounds do not converge to the same value. Figure $3 \mathrm{~b}$ shows that the upper bound is sharp for small values of $c$ and loose for intermediate 
values of $c$ (Figure 3c), and that both the upper and lower bounds converge to 1 as $c$ tends to infinity.

\section{First PAssage times}

Let

$$
W_{n, i}^{c}:=\inf \left\{t \geq 0 ; b_{n}(t)=i\right\}, \quad i=1,2, \ldots
$$

and $\left\{b_{n}^{1}(t) ; W_{n, 1}^{c} \geq t \geq 0\right\}$ be a modified process, where there is an absorbing state at 1, or the ultimate ancestor. The modified process is the same as that introduced for ancestral recombination graph, where $4 c$ is replaced by the recombination parameter $\rho$ (Griffiths (1991)). Theorems 1, 2, 3 in Griffiths (1991) hold for the modified process. The modified process was studied by Krone and Neuhauser (1997). Here, modified processes $\left\{b_{n}^{i}(t) ; W_{n, i}^{c} \geq t \geq 0\right\}$, where there is an absorbing state at $i=1,2, \ldots, n-1$, are studied to discuss the first passage times of the ancestral process $\left\{b_{n}(t) ; t \geq 0\right\}$ at the states $1,2, \ldots, n-1$.

It is possible to show that the expected first passage times of the ancestral process $\left\{b_{n}(t) ; t \geq 0\right\}$ at the states $1,2, \ldots, n-1$ are larger than those in the neutral process $\left\{a_{n}(t) ; t \geq 0\right\} . \mathbb{E}\left[W_{n, 1}^{c}\right]$ is given in Krone and Neuhauser (1997).

Theorem 4.1. Let

$$
W_{n, i}^{0}:=\inf \left\{t \geq 0 ; a_{n}(t)=i\right\}, \quad i=1,2, \ldots
$$

Then,

$$
\mathbb{E}\left[W_{n, i}^{c}\right]=2 \sum_{k=i}^{n-1} \sum_{j=0}^{\infty} \frac{(4 c)^{j}}{(k)_{j+2}}>\mathbb{E}\left[W_{n, i}^{0}\right], \quad i=1,2, \ldots, n-1,
$$

where $W_{n, 1}^{c}$ is the time to the ultimate ancestor. 
Proof. The theorem follows from standard results on birth and death processes (see, e.g., Karlin and Taylor $(1975))$. The modified processes $\left\{b_{n}^{i}(t) ; W_{n, i}^{c} \geq t \geq 0\right\}$ hit the states $i=1,2, \ldots, n-1$ in finite time with probability one, since

$$
\sum_{m=i}^{\infty} \prod_{k=i+1}^{m+1} \frac{\alpha_{k}}{\beta_{k}}=\frac{(4 c)^{i-1}}{(i-1) !} \sum_{m=i}^{\infty} \frac{m !}{(4 c)^{m}}=\infty, \quad i=1,2, \ldots, n-1 .
$$

From the Kolmogorov backward equation for the modified process $\left\{b_{n}^{i}(t) ; W_{n, i}^{c} \geq t \geq 0\right\}$, which is (2.9) for $n=i+1, i+2, \ldots$ with $\xi_{n}=\mathbb{P}\left[b_{n}^{i}(t)=i\right]$ and the boundary condition $\xi_{i}=\delta(t)$, the expected first passage times satisfy a recursion for $i=1,2, \ldots, n-1$

$$
\left(\alpha_{n}+\beta_{n}\right) \zeta(n)-\alpha_{n} \zeta(n-1)-\beta_{n} \zeta(n+1)=1, \quad n=i+1, i+2, \ldots, n-2
$$

with the boundary condition $\zeta(i)=0$, where $\zeta(n)=\mathbb{E}\left[W_{n, i}^{c}\right]$. It is straightforward to solve the recursion and obtain

$$
\mathbb{E}\left[W_{n, i}^{c}\right]=\sum_{m=i}^{\infty} \gamma_{m}+\sum_{j=i}^{n-2} \prod_{k=i+1}^{j+1} \frac{\alpha_{k}}{\beta_{k}} \sum_{l=j+1}^{\infty} \gamma_{l}=2 \sum_{k=i}^{n-1} \sum_{j=0}^{\infty} \frac{(4 c)^{j}}{(k)_{j+2}}, \quad i=1,2, \ldots, n-2
$$

and

$$
\mathbb{E}\left[W_{n, n-1}^{c}\right]=\sum_{m=i}^{\infty} \gamma_{m}=2 \sum_{j=0}^{\infty} \frac{(4 c)^{j}}{(k)_{j+2}}
$$

where

$\gamma_{i}=\frac{1}{\alpha_{i+1}}=\frac{2}{i(i+1)}, \quad \gamma_{m}=\frac{\beta_{i+1} \beta_{i+2} \cdots \beta_{m}}{\alpha_{i+1} \alpha_{i+2} \cdots \alpha_{m} \alpha_{m+1}}=\frac{2(4 c)^{m-i}}{(i)_{m-i+2}}, \quad m=i+1, i+2, \ldots$

It is clear from (4.6) and (4.7) that

$$
\mathbb{E}\left[W_{n, i}^{c}\right]>2 \sum_{k=i}^{n-1} \frac{1}{k(k+1)}=2\left(\frac{1}{i}-\frac{1}{n}\right)=\mathbb{E}\left[W_{n, i}^{0}\right], \quad i=1,2, \ldots, n-1 .
$$

As $c \rightarrow \infty$, for $i=1,2, \ldots, n-1$,

$$
\mathbb{E}\left[W_{n, i}^{c}\right]=2 \sum_{k=i}^{n-1} \frac{1}{k(k+1)} \sum_{j=0}^{\infty} \frac{\left(\frac{4 c}{k+2}\right)^{j}}{\prod_{l=0}^{j-1}\left(1+\frac{l}{k+2}\right)}>2 \sum_{k=i}^{n-1} \frac{e^{\frac{4 c}{k+2}}}{k(k+1)} \rightarrow \infty
$$


Corollary 4.2. For the whole population $(n=\infty)$, the expected first passage times are

$$
\mathbb{E}\left[W_{\infty, i}^{c}\right]=2 \sum_{j=0}^{\infty} \frac{(4 c)^{j}}{(j+1)(i)_{j+1}}, \quad i=1,2, \ldots
$$

Proof. It follows immediately from an identity

$$
\sum_{k=i}^{\infty} \frac{1}{(k)_{j+2}}=\frac{1}{(j+1)(i)_{j+1}}, \quad j=0,1, \ldots
$$

It is straightforward to obtain higher moments of the first passage times of the ancestral process $\left\{b_{n}(t) ; t \geq 0\right\}$ at the states $1,2, \ldots, n-1$ in the same manner. The second moments $\mathbb{E}\left[\left(W_{n, i}^{c}\right)^{2}\right]$ satisfy a recursion

$$
\left(\alpha_{n}+\beta_{n}\right) \zeta(n)-\alpha_{n} \zeta(n-1)-\beta_{n} \zeta(n+1)=2 \mathbb{E}\left[W_{n, i}^{c}\right], \quad n=i+1, i+2, \ldots, n-2
$$

with the boundary condition $\zeta(i)=0$, where $\zeta(n)=\mathbb{E}\left[\left(W_{n, i}^{c}\right)^{2}\right]$. However, there is no simple form for the density as in (1.3). The Laplace transform of the first passage times of the ancestral process satisfy a recursion for $i=1,2, \ldots, n-1$

$$
\left(\lambda+\alpha_{n}+\beta_{n}\right) \zeta(n)-\alpha_{n} \zeta(n-1)-\beta_{n} \zeta(n+1)=0, \quad n=i+1, i+2, \ldots, n-2
$$

with the boundary condition $\zeta(i)=1$, where $\zeta(n)=\mathbb{E}\left[e^{-\lambda W_{n, i}^{c}}\right]$.

The joint probability generating function of $b_{n}^{1}(t)$ and $r_{n}(t)$ satisfies a system of differential equation (2.21) with $\xi_{n}=\mathbb{E}\left[q^{b_{n}^{1}(t)} v^{r_{n}(t)}\right]$. By taking $t=\infty$, we have

$$
0=-\left(\alpha_{n}+\beta_{n}\right) \xi_{n}+\alpha_{n} \xi_{n-1}+v \beta_{n} \xi_{n+1}, \quad n=1,2, . .
$$

with the boundary condition $\xi_{1}=1$, where $\xi_{n}=\mathbb{E}\left[v^{r_{n}(\infty)}\right]$. The form of the probability generating function of $r(\infty)$ is

$$
\mathbb{E}\left[v^{r_{n}(\infty)}\right]=\mathbb{E}\left[\exp \left\{-2 c(1-v) \int_{0}^{W_{n, 1}^{v c}} \tilde{b}_{n}^{1}(u) d u\right\}\right]
$$


while the explicit form of the probability generating function is given by Theorem 5.1 in Ethier and Griffiths (1990), where $\rho$ is replaced by $4 c$, and we have

$$
\mathbb{E}\left[s^{r_{n}(\infty)}\right]=\frac{R_{n}(v)}{R_{1}(v)},
$$

where

$$
\begin{aligned}
R_{n}(v) & =\int_{0}^{1} x^{4 c(1-v)-1}(1-x)^{n-1} e^{-4 c v(1-x)} d x \\
& =\frac{(n-1) !}{(4 c(1-v))_{n}}{ }_{1} F_{1}(n ; 4 c(1-v)+n ;-4 c v) \\
& =\sum_{i=0}^{\infty} \frac{(n+i-1) !(-4 c v)^{i}}{(4 c(1-v)+n)_{n+i} i !} .
\end{aligned}
$$

(4.15) provides a way to compute the expectation of the total length of the edges in the ancestral selection graph in the time interval $\left(0, W_{n, 1}^{c}\right)$, and we have

$$
\mathbb{E}\left[\int_{0}^{W_{n, 1}^{c}} b_{n}^{1}(u) d u\right]=\frac{1}{2 c} \mathbb{E}\left[r_{n}(\infty)\right]=\sum_{k=1}^{\infty}(4 c)^{k-1} \sum_{m=1}^{n-1} \frac{1}{(m)_{k}} .
$$

It is possible to obtain the probability that the modified process $\left\{b_{n}^{1}(t) ; W_{n, 1}^{c} \geq t \geq 1\right\}$ hits the states $n+1, n+2, \ldots$

Theorem 4.3. Let $z(1)=0, z(2)=1$, and

$$
z(j)=1+\frac{\alpha_{2}}{\beta_{2}}+\frac{\alpha_{2} \alpha_{3}}{\alpha_{2} \beta_{3}}+\cdots+\frac{\alpha_{2} \alpha_{3} \cdots \alpha_{j-1}}{\beta_{2} \beta_{3} \cdots \beta_{j-1}}=\sum_{k=0}^{j-2} \frac{k !}{(4 c)^{k}}, \quad j=3,4, \ldots
$$

Then, the probability that the modified process $\left\{b_{n}^{1}(t) ; W_{n, 1}^{c} \geq t \geq 1\right\}$ hits the states $m=$ $n+1, n+2, \ldots$ is

$$
\mathbb{P}\left[W_{n, 1}^{c}>W_{n, m}^{c}\right]=\frac{z(n)}{z(m)}
$$

Proof. The theorem follows from standard results on birth and death processes (see, Karlin and Tavlor $(1975)$, pp. 323). It is straightforward to show that $z\left(b_{n}^{1}(t)\right)$ is a martingale for the modified process. $\min \left\{W_{n, 1}^{c}, W_{n, m}^{c}\right\}$ is a Markov time with respect to the modified process. We apply the optional sampling theorem to conclude that

$$
z(n)=\mathbb{E}\left[b_{n}^{1}\left(\min \left\{W_{n, 1}^{c}, W_{n, m}^{c}\right\}\right)\right]=\mathbb{P}\left[W_{n, 1}^{c}>W_{n, m}^{c}\right] z(m), \quad m=n+1, n+2, \ldots
$$


Remark 4.4. For small $c, \mathbb{P}\left[W_{n, 1}^{c}<W_{n, m}^{c}\right]$ can be expanded into a power series in $c$.

$$
\mathbb{P}\left[W_{n, 1}^{c}>W_{n, m}^{c}\right]=\frac{(4 c)^{m-n}}{[m-2]_{m-n}}+O\left(c^{m-n+1}\right), \quad m=n+1, n+2, \ldots
$$

Figure 4 shows the hitting probability for $n=10$ and 50 as a function of $c$. It can be seen that the hitting probability grows quickly around a critical value of $c$. For $n=50$ and $m=51$, the probability grows linearly until $c$ is smaller than 4.5 (See Remark 4.4). Then it approaches 1 quickly.

\section{TIME TO FIXATION}

In studying evolutionary processes from the standpoint of population genetics, the probability and the time to fixation of a mutant gene play important roles. The expected time to fixation of a mutant gene conditional on fixation was obtained by Kimura and Ohta (1969). Furthermore, Ewens (1973) and Maruyama and Kimura (1974) showed that the expected length of time which it takes for an allele to increase frequency from $q$ to $y(>q)$ on the way to fixation is equal to the expected length of time which the same allele takes when its frequency decrease from $y$ to $q$ on the way to extinction. The time-reversibility property is equivalent to the property that the density of the expected sojourn time does not depend on the sign of the selection coefficient, which was shown by Maruyama (1972). While these results are well known, their interpretation in terms of the ancestral process of the whole population $\left\{b_{\infty}(t) ; t \geq 0\right\}$ are interesting.

The fixation probability was obtained by solving the Kolmogorov backward equation for the Wright-Fisher diffusion with directional selection $\left\{x_{p}(t) ; t \geq 0\right\}$ (Kimura (1957)). The fixation probability of the allele $A_{1}$ is

$$
u_{1}(p)=\frac{1-e^{-4 c p}}{1-e^{-4 c}}
$$


and the fixation probability of the allele $A_{2}$ is $1-u_{1}(p)$. It follows from (1.9) that

$$
1-u_{1}(p)=2\left(1-r^{2}\right) e^{c(r-1)} \sum_{k=0}^{\infty} \frac{V_{1 k}^{(1)}(c, r) V_{1 k}^{(1)}(c, 1)}{2 \lambda_{k}} .
$$

It is possible to obtain the fixation probability from the stationary measure of the ancestral process (3.1). If the allele $A_{2}$ fixes in a population, the ancestral particles of the whole population in infinite time backwards consist of type $A_{2}$ particles only, and we have

$$
\mathbb{E}\left[q^{b_{\infty}(\infty)}\right]=\sum_{i=1}^{\infty} \pi_{i} q^{i}=\frac{e^{4 c q}-1}{e^{4 c}-1}=1-u_{1}(p)
$$

The relationship between the fixation probability and the probability generating function of the stationary measure is a special case of Theorem 2 (f) in Athreya and Swart (2005).

The density of time to fixation of the allele $A_{2}$ conditional on fixation has a genealogical interpretation. Let

$$
T_{0}^{c}:=\inf \left\{t \geq 0 ; y_{q}(t)=1\right\}
$$

Then, it follows from the expression

$$
\mathbb{P}\left[T_{0}^{c}<t \mid T_{0}^{c}<\infty\right]=\frac{\mathbb{E}\left[q^{b_{\infty}(t)}\right]}{1-u_{1}(p)}=\frac{\sum_{i=1}^{\infty} \mathbb{P}\left[b_{\infty}(t)=i\right] q^{i}}{\sum_{i=1}^{\infty} \pi_{i} q^{i}}
$$

that the process of fixation of the allele in a diffusion model, in which the left hand side converges to one as $t \rightarrow \infty$, corresponds to convergence of the distribution of the ancestral process $\mathbb{P}\left[b_{\infty}(t)=i\right]$ to its stationary measure $\pi_{i}$ as $t \rightarrow \infty$.

The expected time to fixation of the allele $A_{2}$ conditional on fixation was obtained by solving the Kolmogorov backward equation (Kimura and Ohta (1969), Maruyama (1972)), and

$$
\mathbb{E}\left[T_{0}^{c} \mid T_{0}^{c}<\infty\right]=\int_{0}^{1} \Phi(q, y) d y
$$


where $\Phi(q, y)$ is the density of the expected sojourn time of the allele $A_{2}$ at frequency $y$ in the path starting from frequency $q$ and going to fixation, and

$$
\begin{aligned}
\Phi(q, y) & =\frac{S(y) S(1-y)}{2 c y(1-y) S(1)}, \quad y>q \\
& =\frac{S(y)}{2 c y(1-y)}\left\{\frac{S(1-y)}{S(1)}-\frac{S(q-y)}{S(q)}\right\}, \quad y<q
\end{aligned}
$$

and $S(y)=\exp (4 c y)-1$. Then,

$$
\mathbb{E}\left[T_{0}^{c} \mid T_{0}^{c}<\infty\right]=\int_{0}^{1} \frac{S(y) S(1-y)}{2 c y(1-y) S(1)} d y-\int_{0}^{q} \frac{S(y) S(q-y)}{2 c y(1-y) S(q)} d y
$$

where

$$
\begin{aligned}
\int_{0}^{1} \frac{S(y) S(1-y)}{2 c y(1-y) S(1)} d y & =\frac{\pi_{1}}{8 c^{2}} \sum_{i=1}^{\infty} \sum_{j=1}^{\infty} \frac{(4 c)^{i+j}}{i ! j !} \int_{0}^{1} y^{i-1}(1-y)^{j-1} d y \\
& =\frac{\pi_{1}}{2 c} \sum_{k=1}^{\infty} \frac{(4 c)^{k}}{(k+1) !} \sum_{i=1}^{k} \frac{1}{i(k-i+1)} \\
& =4 \pi_{1} \sum_{k=0}^{\infty} \frac{H_{k+1}(4 c)^{k}}{(k+2) !}
\end{aligned}
$$

$H_{k}=1+1 / 2+\cdots+1 / k$, and

$$
\begin{aligned}
\int_{0}^{q} \frac{S(y) S(q-y)}{2 c y(1-y) S(q)} d y & =\frac{1}{2 c S(q)} \sum_{i=1}^{\infty} \sum_{j=1}^{\infty} \frac{(4 c)^{i+j}}{i ! j !} \int_{0}^{q} \frac{y^{i-1}(q-y)^{j}}{1-y} d y \\
& =\frac{1}{2 c S(q)} \sum_{i=1}^{\infty} \sum_{j=1}^{\infty} \frac{(4 c q)^{i+j}}{i(i+j) !}{ }_{2} F_{1}(1, i, i+j+1 ; q) \\
& =\frac{1}{2 c S(q)} \sum_{i=1}^{\infty} \sum_{j=1}^{\infty} \frac{(4 c q)^{i+j}}{i(i+j) !} \sum_{k=0}^{\infty} \frac{(i)_{k} q^{k}}{(i+j+1)_{k}}
\end{aligned}
$$

It is possible to obtain the expected time to fixation of the allele $A_{2}$ conditional on fixation from the distribution $b_{\infty}(t)$, and we have

$$
\begin{aligned}
\mathbb{E}\left[T_{0}^{c} \mid T_{0}^{c}<\infty\right] & =\frac{1}{\sum_{i=1}^{\infty} \pi_{i} q^{i}} \int_{0}^{\infty} t \frac{d}{d t}\left[\sum_{i=1}^{\infty}\left\{\mathbb{P}\left[b_{\infty}(t)=i\right]-\pi_{i}\right\} q^{i}\right] d t \\
& =\frac{1}{\sum_{i=1}^{\infty} \pi_{i} q^{i}} \sum_{i=1}^{\infty} q^{i} \int_{0}^{\infty}\left\{\mathbb{P}\left[b_{\infty}(t)=i\right]-\pi_{i}\right\} d t
\end{aligned}
$$


From the two expressions (5.8) and (5.9), an identity at $q=0$ follows immediately.

$$
\sum_{k=0}^{\infty} \frac{V_{1 k}^{(1)}(c,-1) V_{1 k}^{(1)}(c, 1)}{\lambda_{k}^{2} N_{1 k}}=e^{2 c} \pi_{1}^{2} \sum_{k=0}^{\infty} \frac{H_{k+1}(4 c)^{k}}{(k+2) !} .
$$

It is straightforward to obtain similar identities by comparing (5.8) and (5.9) in each power of $q$. Moreover, explicit expression for the higher moments of the time to fixation, conditional on fixation, are available (Maruyama, 1972), and they produce similar identities.

The density of the time to fixation of a single mutant gene conditional on fixation has interesting properties. Let

$$
T_{1}^{c}:=\inf \left\{t \geq 0 ; x_{p}(t)=1\right\}
$$

Then, from a time-reversibility argument on the conditional diffusion process (Ewens (1973), Maruyama and Kimura (1974)), we have

$$
\lim _{q \rightarrow 0} \mathbb{P}\left[T_{0}^{c}<t \mid T_{0}^{c}<\infty\right]=\lim _{p \rightarrow 0} \mathbb{P}\left[T_{1}^{c}<t \mid T_{1}^{c}<\infty\right]=\frac{\mathbb{P}\left[b_{\infty}(t)=1\right]}{\pi_{1}}
$$

The same density holds for a mutant gene of allele $A_{1}$ and a mutant gene of allele $A_{2}$. This property has an intuitive genealogical interpretation. The conditional density is given by the probability of the whole population being descended from a single real ancestral particle. Since there is no variation in the population, selection cannot have an effect on it and consequently, the conditional density should not depend on the allelic type.

Theorem 3.2 gives bounds for the density of the time to fixation of a single mutant gene conditional on the fixation, and

$$
\frac{1}{\pi_{1}}-\frac{\eta(\infty ; c) e^{-\lambda_{0} t}+2 c}{\pi_{1} \lambda_{0}} \leq \lim _{q \rightarrow 0} \mathbb{P}\left[T_{0}^{c}<t \mid T_{0}^{c}<\infty\right] \leq \frac{1}{\pi_{1}}-\frac{\eta(\infty ; c) e^{-\lambda_{0} t}+2 c}{\pi_{1}(\eta(\infty ; c)+2 c)}
$$

The bounds are useless when $c$ is large, since the upper and lower bounds do not converge as $c \rightarrow \infty$. When $c$ is small, the lower bound is sharp. Figure 5a shows the bounds when $c=0.1$. Figure $5 \mathrm{~b}$ gives the conditional density and the derivative of the lower 
bound $\pi_{1}^{-1} \eta(\infty ; c) \exp \left(-\lambda_{0} t\right)$. It can be seen that the tail of the conditional density is well characterised by the largest eigenvalue.

It is worth noting that the identity (5.12) gives the following identity in the distribution $b_{n}(t)$. Its interpretation in terms of the ancestral process $\left\{b_{n}(t) ; t \geq 0\right\}$ is unclear.

Remark 5.1. (5.12) gives

$$
\mathbb{P}\left[b_{\infty}(t)=1\right]=\lim _{n \rightarrow \infty} e^{-4 c} \sum_{k=1}^{n}(-1)^{k+1}\left(\begin{array}{l}
n \\
k
\end{array}\right) \mathbb{E}\left[b_{k}(t)\right]
$$

Proof. (5.12) is equivalent to

$$
\lim _{p \rightarrow 0} \frac{f(p, 1 ; t)}{u_{1}(p)}=\lim _{q \rightarrow 0} \frac{f(p, 0 ; t)}{1-u_{1}(p)}=\frac{\mathbb{P}\left[b_{\infty}(t)=1\right]}{\pi_{1}}
$$

where

$$
\begin{aligned}
\lim _{p \rightarrow 0} \frac{f(p, 1 ; t)}{u_{1}(p)} & =\lim _{p \rightarrow 0} \lim _{n \rightarrow \infty} \frac{\mathbb{E}\left[x_{p}(t)^{n}\right]}{u_{1}(p)}=\lim _{p \rightarrow 0} \lim _{n \rightarrow \infty} \frac{\mathbb{E}\left[\left(1-y_{q}(t)\right)^{n}\right]}{u_{1}(p)} \\
& =\lim _{p \rightarrow 0} \lim _{n \rightarrow \infty} \sum_{k=0}^{n}(-1)^{k}\left(\begin{array}{l}
n \\
k
\end{array}\right) \frac{\mathbb{E}\left[q^{b_{k}(t)}\right]}{u_{1}(p)}=\lim _{n \rightarrow \infty} e^{-4 c} \sum_{k=1}^{n}(-1)^{k+1}\left(\begin{array}{l}
n \\
k
\end{array}\right) \frac{\mathbb{E}\left[b_{k}(t)\right]}{\pi_{1}} .
\end{aligned}
$$

In the neutral Wright-Fisher diffusion, the density of time to fixation of a mutant gene conditional on fixation follows

$$
\lim _{q \rightarrow 0} \mathbb{P}\left[T_{0}^{0}<t \mid T_{0}^{0}<\infty\right]=\mathbb{P}\left[a_{\infty}(t)=1\right]
$$

where $T_{0}^{0}$ is the time to fixation of a mutant gene in the neutral Wright-Fisher diffusion. From (5.8), the expected time to fixation of a mutant gene conditional on fixation has a simple form

$$
\lim _{q \rightarrow 0} \mathbb{E}\left[T_{0}^{c} \mid T_{0}^{c}<\infty\right]=4 \pi_{1} \sum_{j=0}^{\infty} \frac{H_{j+1}(4 c)^{j}}{(j+2) !}<\lim _{q \rightarrow 0} \mathbb{E}\left[T_{0}^{0} \mid T_{0}^{0}<\infty\right]=2
$$

where the inequality holds from the following lemma: 
Lemma 5.2. The density of expected sojourn time of the allele $A_{2}$ at frequency $y$ in the path starting from frequency 0 and going to fixation satisfies

$$
\frac{S(y) S(1-y)}{2 c y(1-y) S(1)}<2, \quad 0<y<1
$$

Proof. The inequality is equivalent to

$$
\frac{e^{4 c y}-1}{y} \frac{e^{4 c(1-y)}-1}{1-y}<4 c\left(e^{4 c}-1\right)
$$

or

$$
\sum_{i=0}^{\infty} \sum_{j=0}^{i} \frac{(4 c)^{i} y^{j}(1-y)^{i-j}}{(j+1) !(i-j+1) !}<\sum_{i=0}^{\infty} \frac{(4 c)^{i}}{(i+1) !}
$$

The inequality follows from an inequality

$$
\sum_{j=0}^{i} \frac{y^{j}(1-y)^{i-j}}{(j+1) !(i-j+1) !}<\frac{1}{(i+1) !} \sum_{j=0}^{i} \frac{i ! y^{j}(1-y)^{i-j}}{j !(i-j) !}=\frac{1}{(i+1) !}, \quad i=0,1, \ldots
$$

As $c$ becomes large, $\mathbb{P}\left[b_{\infty}(t)=1\right]$ decreases, while the expected fixation time of a mutant gene conditional on fixation decreases. It is straightforward to show that the inequality for the expected fixation time (5.18) is equivalent to an inequality

$$
\int_{0}^{\infty}\left\{\frac{\mathbb{P}\left[b_{\infty}(t)=1\right]}{\pi_{1}}-\mathbb{P}\left[a_{\infty}(t)=1\right]\right\} d t>0
$$

In the neutral process, both the density of the waiting time until the ancestral process hits the state 1 and the density of the conditional fixation time are given by the probability that the ancestral process is at the state 1 (1.315.17). It follows that

$$
\mathbb{E}\left[W_{\infty, 1}^{0}\right]=\lim _{q \rightarrow 0} \mathbb{E}\left[T_{0}^{0} \mid T_{0}^{0}<\infty\right]=\int_{0}^{\infty}\left\{\mathbb{P}\left[a_{\infty}(t)=1\right]-1\right\} d t=2
$$

In contrast, in the processes with directional selection, we have

$$
\mathbb{E}\left[W_{\infty, 1}^{c}\right]=2 \sum_{j=0}^{\infty} \frac{(4 c)^{j}}{(j+1)(j+1) !}>2
$$


while

$$
\lim _{q \rightarrow 0} \mathbb{E}\left[T_{0}^{c} \mid T_{0}^{c}<\infty\right]=\int_{0}^{\infty}\left\{\frac{\mathbb{P}\left[b_{\infty}(t)=1\right]}{\pi_{1}}-1\right\} d t=4 \pi_{1} \sum_{j=0}^{\infty} \frac{H_{j+1}(4 c)^{j}}{(j+2) !}<2 .
$$

\section{DISCUSSION}

In this article, the ancestral process $\left\{b_{n}(t) ; t \geq 0\right\}$, specifying the number of branches in the ancestral selection graph, was investigated by exploiting the moment duality between this process and the Wright-Fisher diffusion with directional selection. An explicit formula for the probability distribution of $b_{n}(t)$ was derived. Although this expression cannot be given in closed form, since it involves eigenvalues and coefficients which are determined by an intractable three-term recursion relation, it is possible to expand the probability distribution as a perturbation series in $2 c$. This expression is given in a closed form for each order of the perturbation and is accurate when $c$ is small. Bounds for the probability that $b_{n}(t)$ is at the state 1 is obtained. When $c$ is small, one of the bounds is sharp. The density of time to fixation of a single mutant gene conditional on fixation was shown to be given by the probability of the whole population being descended from a single real particle, regardless of the allelic type. Thus, the bounds for the probability that $b_{n}(t)$ is at the state 1 give bounds for the density of the conditional hitting time. It was shown that the tail of the conditional hitting time is well characterised by the largest eigenvalue. The probability that the process hits states larger than the initial state before the process hits the state 1 was obtained. According to the formula, the number of branches in the ancestral selection graph grows rapidly when $c$ is larger than a critical value. One of the difficulties of simulating the ancestral selection graph is keeping track of large numbers of branches when $c$ is large. Specifically, when $c$ is larger than the critical value, enormous number of branches emerge and it will be difficult to simulate the ancestral selection graph.

If a sample consists only of type $A_{2}$ particles, the probability distribution of the ancestral particles, all of which are $A_{2}$, is $b_{n}(t)$ (see Theorem 2.1). If a sample contains type $A_{1}$ particles, the joint probability distribution of the number of the $A_{1}$ particles and 
the number of the $A_{2}$ particles is interesting. However, it seems that the expression of the moments in the Wright-Fisher diffusion (2.7) does not give any insights into the joint probability distribution, except in the case when a sample consists of a single $A_{1}$ particle. The time-reversibility argument of the conditional diffusion process gives an identity, whose interpretation in terms of the ancestral process is unclear (see Remark 5.1). The interpretation of the time-reversibility in terms of the ancestral process needs further investigation.

In this article, the fixation process of a mutant gene in the Wright-Fisher diffusion with directional selection, which has been well studied, was interpreted in terms of the convergence of the ancestral process to its stationary measure. This approach might be useful for other population genetical models for which less is known about the fixation process. Examples include diffusion processes with frequency-dependent selection, with multiple selected alleles, and of spatially-structured populations (Athreva and Swart, 2005).

\section{Appendix A. The oblate spheroidal Wave FunCtion}

The oblate spheroidal wave function $V_{1 k}^{(1)}(c, z)$ can be represented by expansions of the form (Stratton et al. (1941))

$$
V_{1 k}^{(1)}(c, z)=\sum_{l \geq 0}{ }^{\prime} f_{l}^{k}(c) T_{l}^{1}(z), \quad k=0,1, \ldots
$$

This notation was used in Kimura (1955). It was denoted by $V_{1 k}^{(1)}(-i c, z)$ in Stratton et al. (1941) and $\left(1-z^{2}\right)^{\frac{1}{2}} S_{1 k+1}(c, z)$ in Flammer (1957). From the orthogonal properties of the Gegenbauer function it is shown that

$$
\int_{-1}^{1}\left(1-z^{2}\right) V_{1 k}^{(1)}(c, z) V_{1 l}^{(1)}(c, z) d z=\delta_{k, l} N_{1 k}
$$

where

$$
N_{1 k}=2 \sum_{l \geq 0} \frac{(l+1)(l+2)}{(2 l+3)}\left(f_{l}^{k}(c)\right)^{2}
$$


Note that

$$
V_{1 k}^{(1)}(c, 1)=\frac{1}{2} \sum_{l \geq 0}^{\prime}(l+1)(l+2) f_{l}^{k}(c), \quad V_{1 k}^{(1)}(c,-1)=(-1)^{k} V_{1 k}^{(1)}(c, 1) .
$$

The coefficients $f_{l}^{k}(c)$ satisfy a three-term recursion in the form

$$
A_{l+2} f_{l+2}^{k}(c)+B_{l} f_{l}^{k}(c)+C_{l-2} f_{l-2}^{k}(c)=0,
$$

where

$$
A_{l}=-\frac{(l+1)(l+2)}{(2 l+1)(2 l+3)}, \quad B_{l}=\frac{l(l+3)-b_{k}}{c^{2}}-\frac{2 l^{2}+6 l+1}{(2 l+1)(2 l+5)}, \quad C_{l}=-\frac{(l+1)(l+2)}{(2 l+3)(2 l+5)},
$$

and $b_{k}=2 \lambda_{k}-2-c^{2} \cdot f_{l}^{k}(c)=0$ for odd $l$ if $k$ is even and for even $l$ if $k$ is odd. (A.4) can be developed as a continued fraction.

$$
\begin{aligned}
\frac{f_{l}^{k}}{f_{l+2}^{k}}= & -\frac{A_{l+2}}{B_{l}-} \frac{C_{l-2} A_{l}}{B_{l-2}-} \cdots \frac{C_{2} A_{4}}{B_{2}-} \frac{A_{2}}{B_{0}} \quad l=0,2, \ldots \\
& -\frac{A_{l+2}}{B_{l}-} \frac{C_{l-2} A_{l}}{B_{l-2}-} \cdots \frac{C_{3} A_{5}}{B_{3}-} \frac{A_{3}}{B_{1}} \quad l=1,3, \ldots
\end{aligned}
$$

and

$$
\frac{f_{l+2}^{k}}{f_{l}^{k}}=-\frac{C_{l}}{B_{l+2}-} \frac{A_{l+4} C_{l+2}}{B_{l+4}-} \cdots, \quad l=0,1, . .
$$

$b_{k}$ is determined by the condition that the reciprocal of the ratio $f_{l} / f_{l+2}$ by (A.5) must equal the value of $f_{l+2} / f_{l}$ obtained from (A.6). Then, the continued fractions provide a way to compute arbitrary coefficient.

For small $c$, the eigenvalue can be expanded into a power series in $c$.

$$
\lambda_{k}=\frac{(k+1)(k+2)}{2}+\frac{(k+1)(k+2)}{(2 k+1)(2 k+5)} c^{2}+O\left(c^{4}\right) .
$$

If we set $f_{k}^{k}(c)=1$, then

$$
f_{k+2}^{k}(c)=\frac{(k+1)(k+2)}{2(2 k+3)(2 k+5)^{2}} c^{2}+O\left(c^{4}\right), \quad f_{k-2}^{k}(c)=-\frac{(k+1)(k+2)}{2(2 k+1)^{2}(2 k+3)} c^{2}+O\left(c^{4}\right),
$$

and other coefficients are zero up to $O\left(c^{4}\right)$. 
Acknowledgments. The author is grateful for useful discussion with Jotun Hein, his group, Robert C. Griffiths and Jay E. Taylor. He is also grateful for valuable comments and suggestions by anonymous referees.

\section{REFERENCES}

Athreya, S. R. and Swart, J. M. (2005) Branching-coalescing particle systems. Prob. Theory Relat. Fields 131 376-414.

Erdélyi, A. Ed. (1954). Tables of Integral Transforms, Vol. II. McGraw-Hill, New York.

Ethier, S. N. and Griffiths, R. C. (1990). On the two-locus sampling distribution. J. Math. Biol. 29 131-159.

Ethier, S. N. and Kurtz, T. G. (1986) Markov Processes: Characterization and Convergence. Jonh Wiley \& Sons, New York.

Ewens, W. J. (1973). Conditional diffusion process in population genetics. Theor. Pop. Biol. 4 21-30.

Fearnhead, P. (2002) The common ancestor at a nonneutral locus. J. Appl. Prob. 39 38-54.

Flammer, C. (1957). Spheroidal Wave Functions. Stanford University Press, Stanford.

Gantmacher, F. R. (1959). Matrix Theory, Vol. II. Chelsea, New York.

Griffiths, R. C. (1979). Exact sampling distributions from the infinite neutral allele model. Adv. Appl. Prob. 11 326-354.

Griffiths, R. C. (1980). Line of descent in the diffusion approximation of neutral WrightFisher models. Theor. Pop. Biol. 17 37-50.

Griffiths, R. C. (1991). The two-locus ancestral graph, in "Selected Proceedings of the Symposium on Applied Probability, Sheffield, 1989" (I. V. Basawa and R. L. Taylor, Eds.), pp. 100-117, IMS Lecture Notes-Monograph Series, Vol. 18, Institute of Mathematical Statistics.

Karlin, S. and Taylor, H. M. (1975). A First Course in Stochastic Processes, 2nd. ed. Academic Press, San Diego. 
Kimura, M. (1955). Stochastic process and distribution of gene frequencies under natural selection. Cold Spring Harbor Symposia on Quantitative Biology 20 33-53.

Kimura, M. (1957). Some problems of stochastic processes in genetics. Ann. Math. Stat. $28882-901$.

Kimura, M. and Ohta, T. (1969) The average number of generations until fixation of a mutant gene in a finite population. Genetics 61 763-771.

Kingman, J. F. C. (1982). On the genealogy of large populations. J. Appl. Probab. 19 $27-43$.

Kingman, J. F. C. (1982). The coalescent. Stochastic Process. Appl. 13 235-248.

Krone, S. M. and Neuhauser, C. (1997). Ancestral process with selection. Theor. Pop. Biol. $51210-237$.

Maruyama, T. (1972). The average number and the variance of generations at particular gene frequency in the course of fixation of a mutant gene in a finite population. Genet. Res. Camb. 19 109-113.

Maruyama, T. and Kimura, M. (1974). A note on the speed of gene frequency changes in reverse directions in a finite population. Evolution 28 161-163.

Shiga, T. (1981) Diffusion processes in population genetics. J. Math. Kyoto Univ. 21 $133-151$.

Stratton, J. A., Morse, P. M. Chu, L. J. and Hunter, R. A. (1941). Elliptic Cylinder and Spheroidal Wave Functions. John Wiley and Sons, New York.

Tavaré, S. (1984). Line-of-descent and genealogical processes, and their applications in population genetics. Theor. Pop. Biol. 26 119-164. 
Figure 1. A realization of the ancestral selection graph embedded in a diagram of a sample path of $x_{p}(t)$.

Figure 2. $\eta(\infty ; c)($ dots $)$ and $3 \exp (-c)$ (line).

Figure 3. $1-\mathbb{P}\left[b_{10}(t)=1\right]$ (line) and the upper and the lower bounds given by Theorem 3.2 (dotted lines).

Figure 4. The probability that the ancestral process $\left\{b_{n}(t) ; t \geq 0\right\}$ hits states $m(>n)$ before the process hits state 1 (ultimate ancestor).

Figure 5. (a) The cumulative probability of the density of time to fixation of a single mutant gene conditional on the fixation (5.12) (line) and the upper and the lower bounds given by (5.13) (dotted lines). (b) The density of the conditional fixation time (line) and $\pi_{1}^{-1} \eta(\infty ; c) \exp \left(-\lambda_{0} t\right)$ (dotted line). 


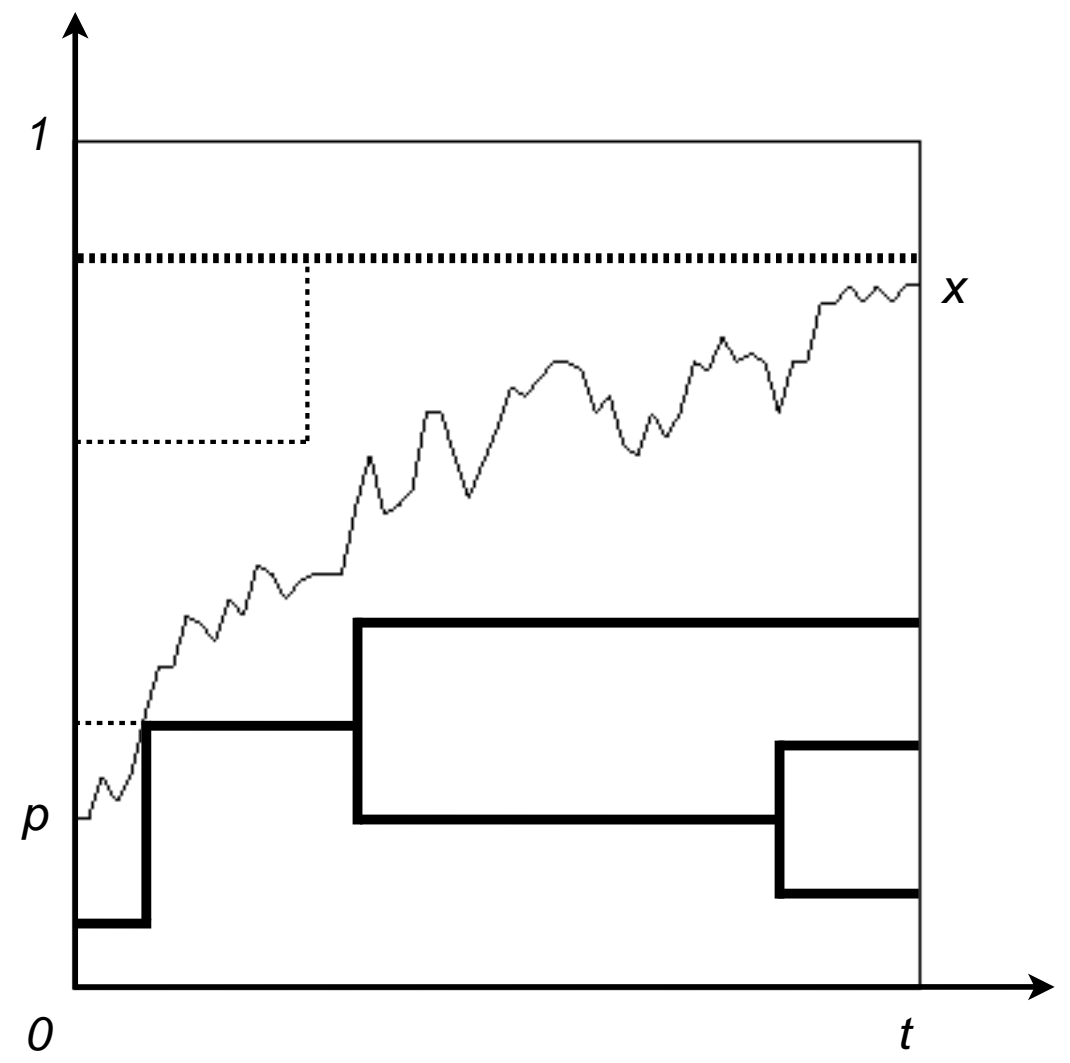

Figure 1. 


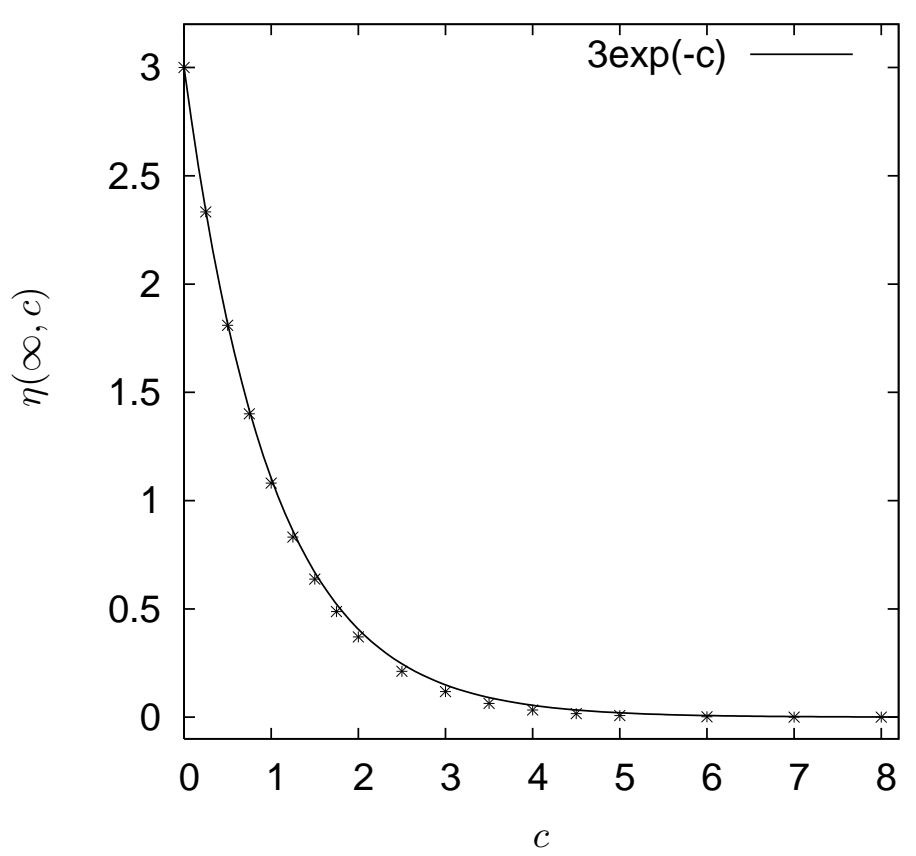

Figure 2. 
(a) $\mathrm{C}=0$

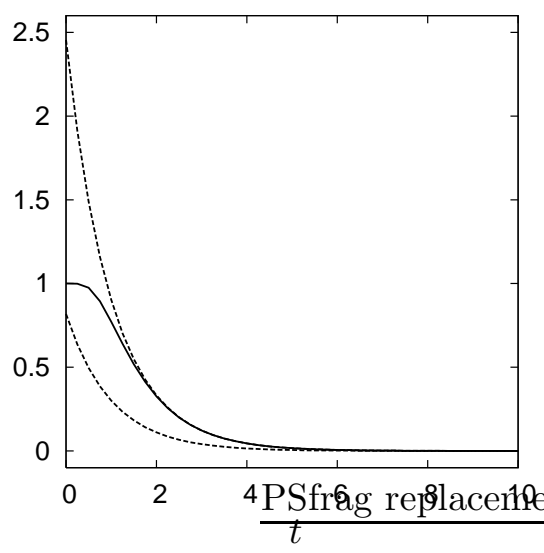

(c) $\mathrm{C}=1$

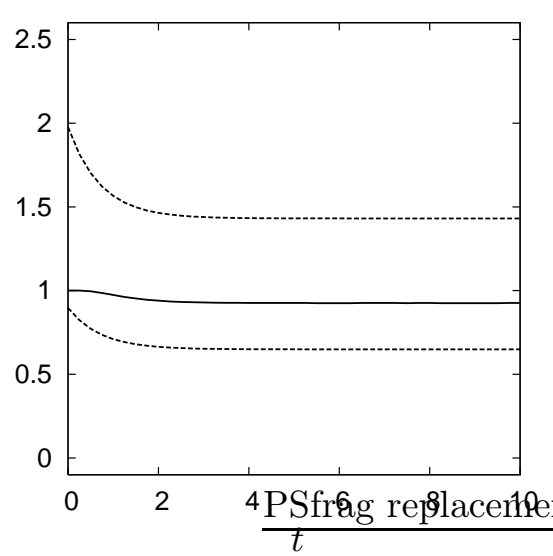

(b) $\mathrm{c}=0.1$

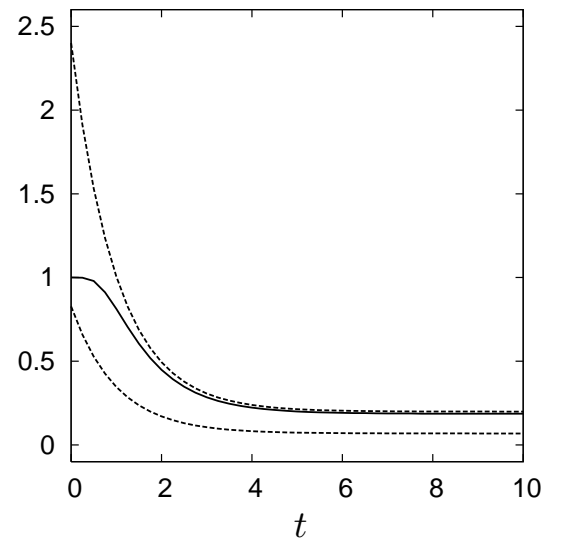

(d) $\mathrm{C}=8$

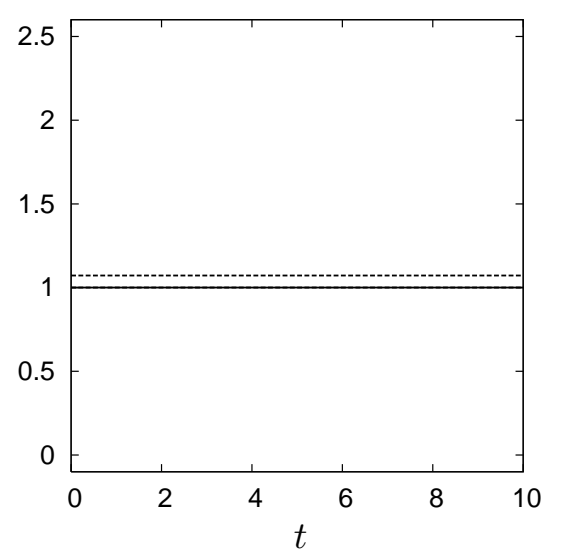

Figure 3. 
(a) $n=10$

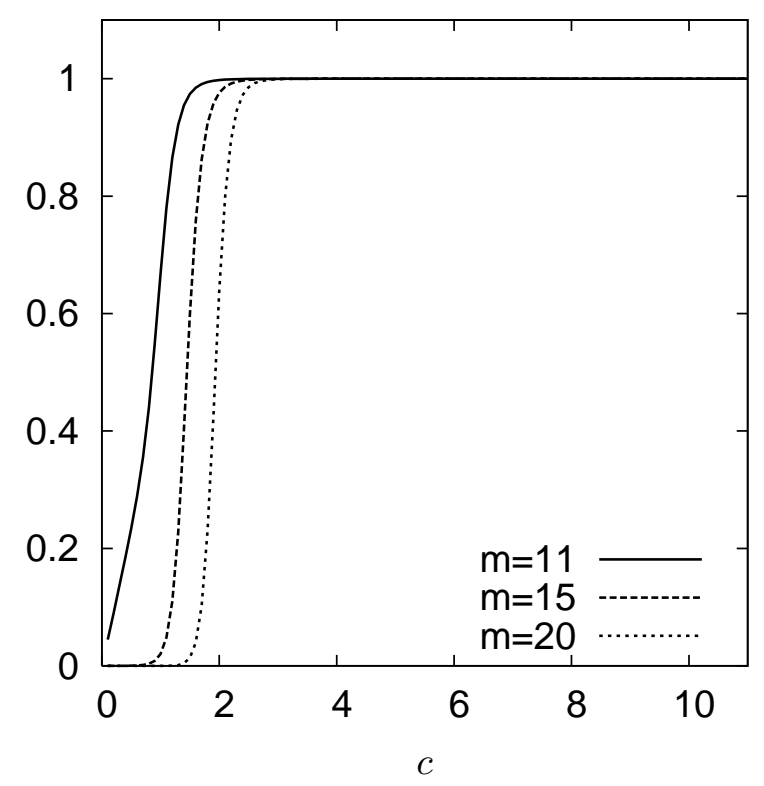

(b) $n=50$

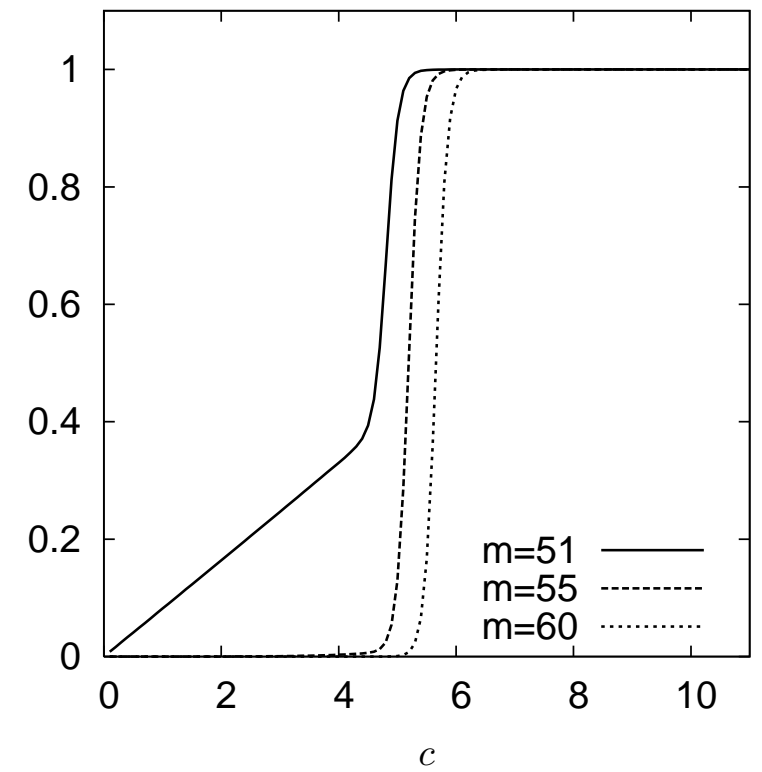

Figure 4. 
(a)

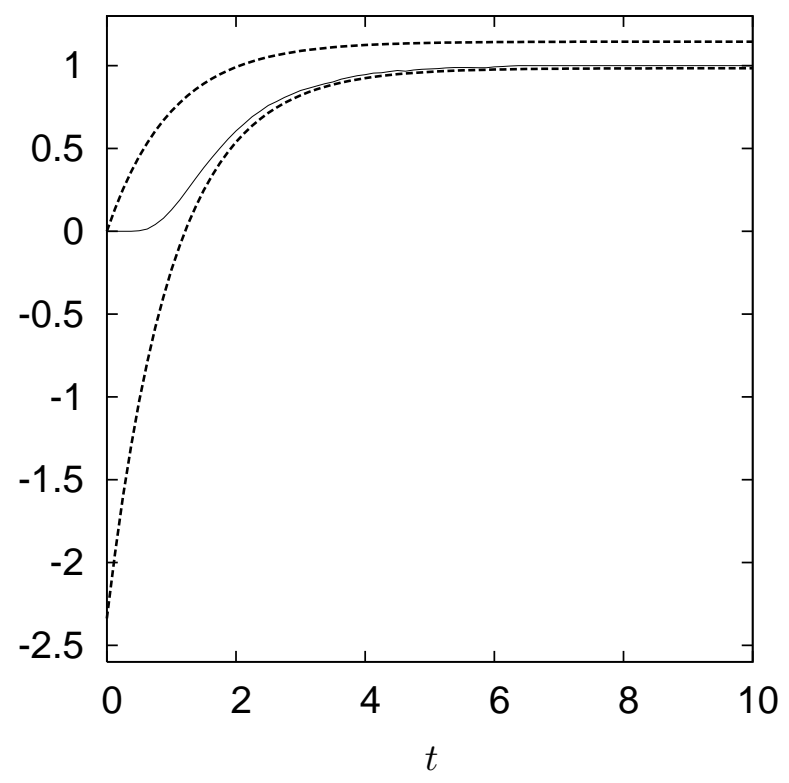

(b)

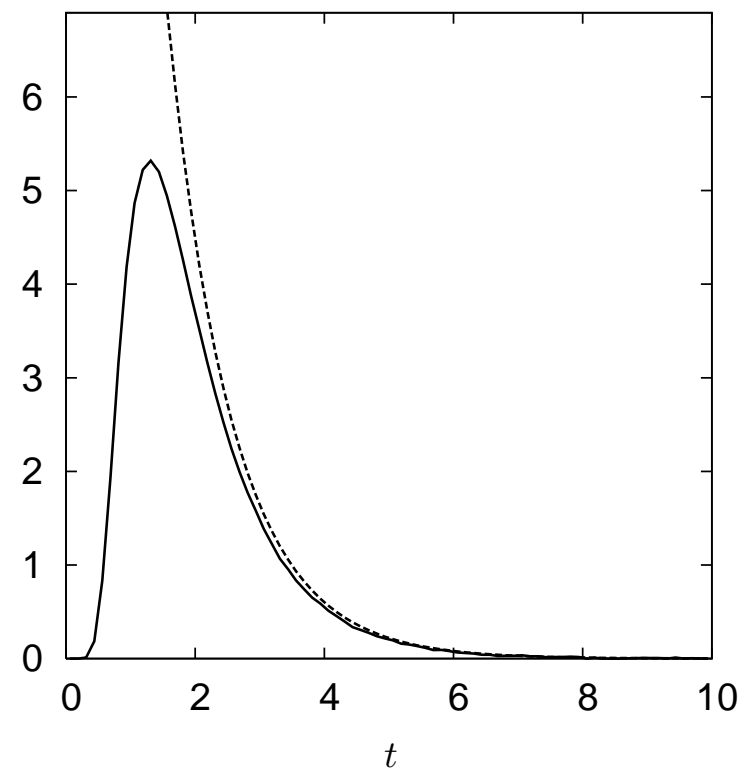

Figure 5. 